Erratum

\title{
Erratum to "Development of Allometric Equations for Estimating Above-Ground Liana Biomass in Tropical Primary and Secondary Forests, Malaysia"
}

\author{
Patrick Addo-Fordjour ${ }^{1,2}$ and Zakaria B. Rahmad ${ }^{1}$ \\ ${ }^{1}$ School of Biological Sciences, University of Science Malaysia, 11800 Pulau Penang, Penang, Malaysia \\ ${ }^{2}$ Department of Theoretical and Applied Biology, College of Science, Kwame Nkrumah University of Science and Technology (KNUST), \\ Kumasi, Ghana \\ Correspondence should be addressed to Patrick Addo-Fordjour; paddykay77@yahoo.com \\ Received 19 June 2013; Accepted 12 August 2013 \\ Copyright (c) 2013 P. Addo-Fordjour and Z. B. Rahmad. This is an open access article distributed under the Creative Commons \\ Attribution License, which permits unrestricted use, distribution, and reproduction in any medium, provided the original work is \\ properly cited.
}

In Table 4 values of $\beta( \pm$ SE) are modified from $0.394 \pm 0.010$ to $0.394 \pm 0.202$ in the 3 rd row and from $0.431 \pm 0.062$ to $0.431 \pm 0.423$ in the 7 th row.

In Table 5 values of $\beta( \pm$ SE) are modified from $0.450 \pm$ 0.010 to $0.450 \pm 0.223$ in the 3 rd row and from $0.452 \pm 0.044$ to $0.452 \pm 0.551$ in the 7 th row. 
TABLE 4: Allometric equations of mixed species for estimating liana stem biomass ( $\mathrm{kg}$ ).

\begin{tabular}{|c|c|c|c|c|c|c|}
\hline \# & Equation & $c( \pm \mathrm{SE})$ & $\alpha( \pm \mathrm{SE})$ & $\beta( \pm \mathrm{SE})$ & $R^{2}$ (adjusted) & FI \\
\hline 1 & Stem biomass $=c+\alpha D$ & $0.154 \pm 0.078$ & $1.731 \pm 0.028$ & - & 0.989 & 0.58 \\
\hline 2 & Stem biomass $=c+\alpha L$ & $2.550 \pm 0.189$ & $0.416 \pm 0.010$ & - & 0.896 & 1.70 \\
\hline 3 & Stem biomass $=c+\alpha D+\beta L$ & $0.474 \pm 0.258$ & $0.452 \pm 0.037$ & $0.394 \pm 0.202$ & 0.976 & 0.84 \\
\hline 4 & $(\text { Stem biomass })^{0.9}=c+\alpha D$ & $0.782 \pm 0.098$ & $1.210 \pm 0.015$ & - & 0.990 & 0.53 \\
\hline 5 & Stem biomass $=c+\alpha D^{2}$ & $0.144 \pm 0.089$ & $0.349 \pm 0.005$ & - & 0.861 & 2.63 \\
\hline 6 & $\log _{10}($ Stem biomass $)=c+\alpha\left(\log _{10} D\right)$ & $0.396 \pm 0.0033$ & $1.086 \pm 0.042$ & - & 0.981 & 0.18 \\
\hline 7 & $\log _{10}($ Stem biomass $)=c+\alpha\left(\log _{10} D\right)+\beta\left(\log _{10} L\right)$ & $0.165 \pm 0.002$ & $0.432 \pm 0.063$ & $0.431 \pm 0.423$ & 0.954 & 0.47 \\
\hline
\end{tabular}

\#: equation number; $D$ : liana diameter; $L$ : liana length.

TABLE 5: Allometric equations of mixed species for estimating total above-ground biomass (kg) of lianas.

\begin{tabular}{|c|c|c|c|c|c|c|}
\hline \# & Equation & $c( \pm \mathrm{SE})$ & $\alpha( \pm \mathrm{SE})$ & $\beta( \pm \mathrm{SE})$ & $R^{2}$ (adjusted) & FI \\
\hline 8 & Total biomass $=c+\alpha D$ & $0.262 \pm 0.181$ & $1.934 \pm 0.029$ & - & 0.992 & 0.58 \\
\hline 9 & Total biomass $=c+\alpha L$ & $3.120 \pm 0.413$ & $0.476 \pm 0.021$ & - & 0.900 & 1.92 \\
\hline 10 & Total biomass $=c+\alpha D+\beta L$ & $0.768 \pm 0.280$ & $0.511 \pm 0.040$ & $0.450 \pm 0.223$ & 0.987 & 0.89 \\
\hline 11 & $(\text { Total biomass })^{0.9}=c+\alpha D$ & $1.041 \pm 0.096$ & $1.354 \pm 0.015$ & - & 0.993 & 0.53 \\
\hline 12 & Total biomass $=c+\alpha D^{2}$ & $0.194 \pm 0.041$ & $0.405 \pm 0.007$ & - & 0.904 & 2.41 \\
\hline 13 & $\log _{10}($ Total biomass $)=c+\alpha\left(\log _{10} D\right)$ & $0.490 \pm 0.021$ & $1.090 \pm 0.027$ & - & 0.986 & 0.22 \\
\hline 14 & $\log _{10}($ Total biomass $)=c+\alpha\left(\log _{10} D\right)+\beta\left(\log _{10} L\right)$ & $0.275 \pm 0.021$ & $0.470 \pm 0.066$ & $0.452 \pm 0.551$ & 0.960 & 0.49 \\
\hline
\end{tabular}

\#: equation number; $D$ : liana diameter; $L$ : liana length. 

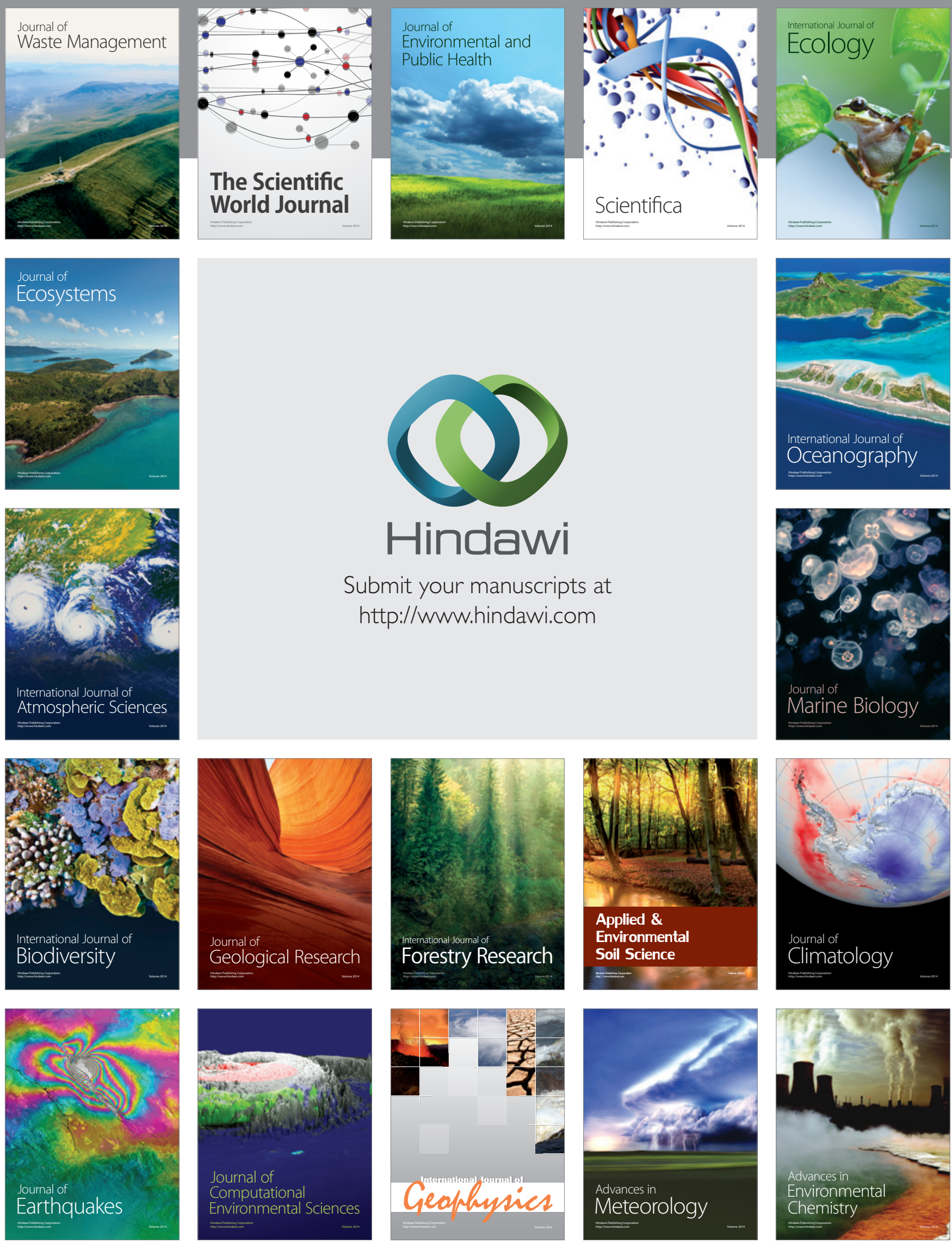